\title{
Structure of the photospheric magnetic field during sector crossings of the heliospheric magnetic field
}

\author{
Tibebu Getachew ${ }^{1} \cdot$ Ilpo Virtanen $^{1} \cdot$ Kalevi Mursula $^{1}$
}

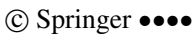

\begin{abstract}
The photospheric magnetic field is the source of the coronal and heliospheric magnetic fields (HMF), but their mutual correspondence is non-trivial and depends on the phase of the solar cycle. The photospheric field during the HMF sector crossings observed at $1 \mathrm{AU}$ has been found to contain enhanced field intensities and definite polarity ordering, forming regions called Hale boundaries. Here we study the structure of the photospheric field during the HMF sector crossings during solar cycles 21-24, separately for the four phases of each solar cycle. We use a refined version of Svalgaard's list of major HMF sector crossings, mapped to the Sun using the solar wind speed observed at the Earth, and the daily level-3 magnetograms of the photospheric field measured at the Wilcox Solar Observatory in 1976-2016. We find that the structure of the photospheric field corresponding to the HMF sector crossings, and the existence and properties of the corresponding Hale bipolar regions varies significantly with solar cycle, solar cycle phase and hemisphere. The Hale boundaries in more than half of the ascending, maximum and declining phases are clear and statistically significant. The most clear Hale boundaries are found during the $(+,-)$ HMF crossings in the northern hemisphere of odd cycles 21 and 23, but less systematical during the (+,-) crossings in the southern hemisphere of even cycles 22 and 24 . No similar difference between odd and even cycles is found for the $(-,+)$ crossings. Thus, overall, the northern hemisphere has a more organized Hale pattern. The photospheric field distribution also depicts a larger area for the field of the northern hemisphere during the declining and minimum phases, in a good agreement with the bashful ballerina phenomenon.
\end{abstract}

Keywords: Sun: photosphere, Sun: magnetic fields, Sun: activity, Sun: heliosphere, Sun: solar wind

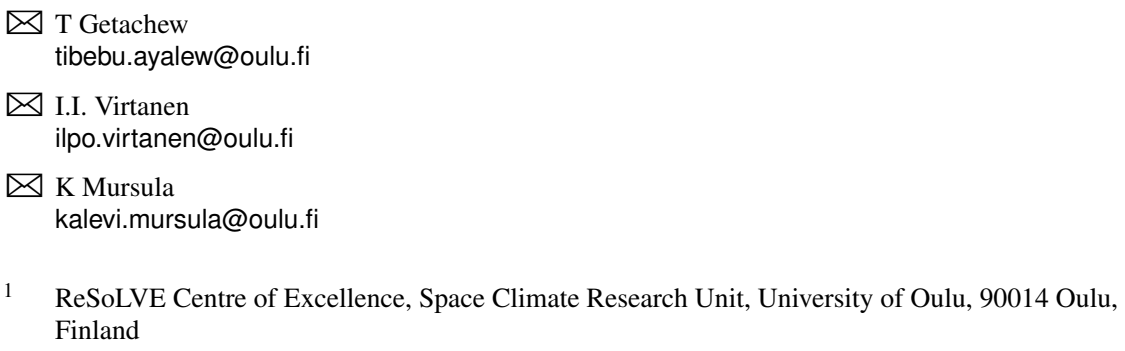




\section{Introduction}

The heliospheric magnetic field (HMF) is organized into sectors of polarities oriented predominantly away or toward the Sun (Wilcox and Ness, 1965). The heliospheric current sheet (HCS), which is the extension of solar magnetic equator in space, separates the two sectors. Because of the tilt of the Sun's magnetic dipole with respect to the rotation axis, the HCS makes a flapping surface also called the ballerina skirt (Smith et al., 2000). The structure of the HCS has been found to greatly vary over the course of a solar cycle (SC). Several studies have shown the momentary (Simpson, Zhang, and Bame, 1996; Crooker et al., 1997; Smith et al., 2000), or the persistent (Mursula and Hiltula, 2003; Erdös and Balogh, 2010; Virtanen and Mursula, 2010; McIntosh et al., 2013) hemispheric asymmetry of the HCS. The southward shift of the HCS is found to be a persistent pattern during roughly three years of the declining phase of all studied solar cycles, a phenomenon also called the bashful ballerina (Mursula and Hiltula, 2003). Hemispheric asymmetry of magnetic activity has also been found in the corona and photosphere (Zhao, Hoeksema, and Scherrer, 2005; Norton and Gallagher, 2010; Wang and Robbrecht, 2011; McIntosh et al., 2013; Virtanen and Mursula, 2014), and is known to be an essential property of the solar dynamo (Mursula and Hiltula, 2004; Hiltula and Mursula, 2006; Norton, Charbonneau, and Passos, 2014).

The number of HMF sectors in the ecliptic plane varies in time. Depending on the solar cycle phase, mostly two or four sectors per solar rotation are observed in the ecliptic at 1AU. It has long been known that the two-sector structure is the result of the tilted dipole and most commonly observed in the late declining to minimum phase of a solar cycle, whereas the four-sector structure is formed by an additional equatorial quadrupole contribution to the main dipole and typically seen during the maximum phase (Schulz, 1973; Hoeksema, Wilcox, and Scherrer, 1983; Sanderson et al., 2003; Balogh and Erdös, 2013). However, at times like during the declining phase of cycle 23 , when the dipole is relatively weak compared to the quadrupole term, the foursector structure is also more abundant. Due to the unipolar magnetic fields of polar coronal holes, one HMF sector is dominating in north and the other in south around solar minima (Rosenberg and Coleman, 1969). Depending on solar polarity, one of the two sectors of the HMF dominates in Fall (Spring) when the Earth achieves its highest northern (southern) heliographic latitudes due to the $7.2^{\circ}$ tilt of the solar rotation axis with respect to the ecliptic. In the declining phase when the HCS is shifted southward, the dominance of the HMF sector of the northern hemisphere is systematically more strongly represented at $1 \mathrm{AU}$ and elsewhere in the low-latitude heliosphere, irrespective of solar magnetic polarity (Mursula and Hiltula, 2003; Mursula and Virtanen, 2011). On the other hand, the streamer belt is found to be systematically shifted toward the northern magnetic hemisphere during declining and solar minimum times (Crooker et al., 1997; Zieger and Mursula, 1998; Mursula and Zieger, 2001; Mursula, Hiltula, and Zieger, 2002).

The HMF sector structure reflects the large-scale magnetic field of the solar corona (Antonucci and Svalgaard, 1974), which has its origin in the underlying photosphere (Wilcox and Howard, 1968; Svalgaard, Hannah, and Hudson, 2011). Recent studies have shown that the photospheric magnetic field has a maximum near a Hale boundary, a sector boundary where the change of sector polarity follows the Hale polarity rule for sunspots of the current cycle and hemisphere (Svalgaard and Wilcox, 1976; Svalgaard, 
Hannah, and Hudson, 2011; Hudson, Svalgaard, and Hannah, 2014). The warps in $\mathrm{HCS}$, for example at $1 \mathrm{AU}$, are assumed to predominantly originate from these Hale boundaries (Svalgaard, Hannah, and Hudson, 2011). It has been also shown that the HMF sector structure is associated with highly concentrated fields of sunspots (Hudson, Svalgaard, and Hannah, 2014) and solar flares (Dittmer, 1975; Svalgaard, Hannah, and Hudson, 2011; Hudson, Svalgaard, and Hannah, 2014) and the association is stronger at the Hale boundary.

In this paper we study the structure of the photospheric magnetic field around HMF sector crossings, i.e., at times when the HMF sector is seen to change polarity at the Earth. The sector crossing observed at the Earth is mapped back to the photosphere to get the corresponding photospheric field structure at the expected source. We use 40 years of daily magnetograms measured at the Wilcox solar observatory from 1976-2016 to analyze the photospheric magnetic field separately for different solar cycles and cycle phases. The paper is organized as follows. Section 2 presents the data and methods used. Sections from 3 - 6 focus on the results obtained for solar cycles 21-24, respectively. Section 7 focuses on the statistical estimate of the significance of the observed Hale boundaries. Discussion and conclusions are presented in Section 8.

\section{Data and methods}

\subsection{HMF sector definition}

Based on observations of polar geomagnetic data, Svalgaard (1968) and Mansurov (1970) showed that the dominant daily HMF sector can be determined from the diurnal variation of the high latitude geomagnetic field. This is commonly called the SvalgaardMansurov effect. Recently, Vokhmyanin and Ponyavin (2013) used this effect to study the HMF sector occurrence more than a century ago. Svalgaard has prepared a list of HMF sectors and sector crossing times (sector boundaries) at daily resolution. The list is derived from geomagnetic and satellite HMF data. When satellite HMF data are available they are used as the main source to calculate sector crossing times, while the data gaps are filled using geomagnetic data. For modern times (since 1970s), the satellite HMF data are the dominant source. He defined sector crossing times based on the criteria that a well-defined sector boundary has at least four days of same polarity on either side of the boundary. Here we used this sector crossing list from 1976-2016 as one definition of HMF sector crossings and assigned the sector crossing times at the beginning of the first new polarity UT day. (The list as well as details of the compilation method can be found at http://wso.stanford.edu/). The HMF sectors according to Svalgaard's list are shown in the second panel of Figure 1 as a function of Carrington longitude and time.

In this paper, we sought to improve the daily resolution of Svalgaard's sector crossing times by studying the hourly HMF polarities using the OMNI data set (solar wind and HMF data was retrieved from http://omniweb.gsfc.nasa.gov/). We use the plane division ( $B_{x}-B_{y}<0$ away sector and $B_{x}-B_{y}>0$ towards sector) to define the sectors and the HMF polarity. The top panel of Figure 1 shows the HMF sector structure for the 

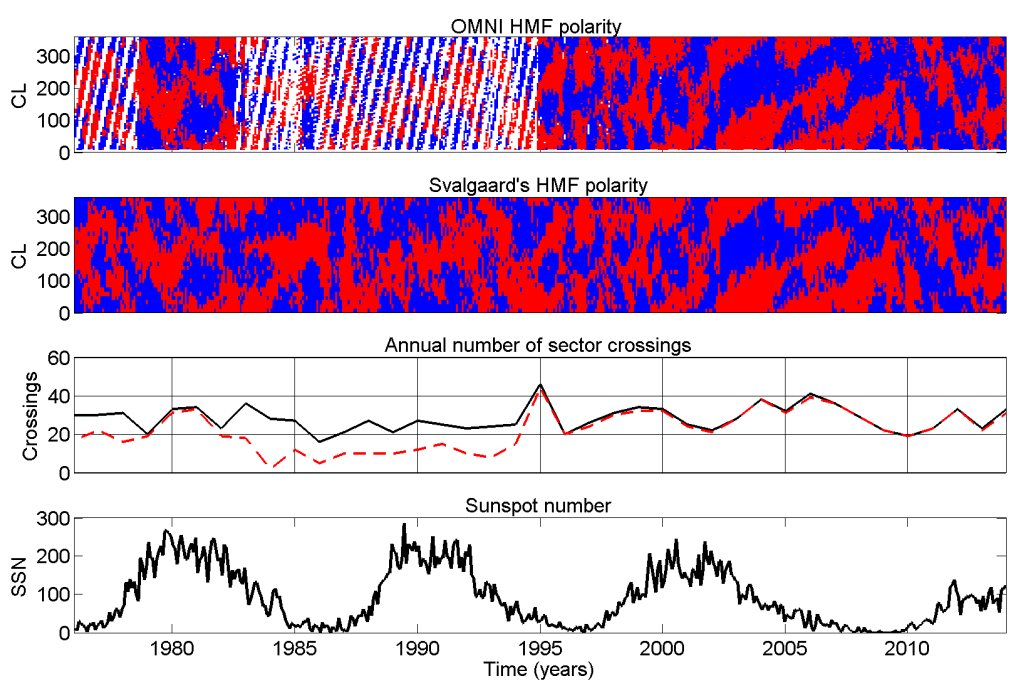

Figure 1. Panels, from top to bottom: Top: HMF polarity of OMNI data base with time and Carrington longitude (CL); Second: same for Svalgaard's list; Third: annual number of Svalgaard's sector crossings (refined HMF sector crossings, respectively) indicated by black solid (red dashed) line; Bottom: the 13-month running mean sunspot number obtained from SIDC. In the top and second panels, the away (toward) sector is indicated by the red (blue) color.

OMNI data in 1976-2016. The sector structure is presented using hourly satellite observations smoothed with a 13-hour running mean (with $40 \%$ data coverage required for each hourly mean). We use this 13-hour running mean method also to find the improved sector crossing times within \pm 31 hours from Svalgaard's sector crossing times. (Note that 31 is the sum of one day and half the filter length of $7 h$ ). If more than one sector crossing is obtained within this window, the one nearest to Svalgaard's sector crossing time with the same order of polarities is selected. If no sector crossing is found, e.g., due to a gap in OMNI data, we ignore that sector crossing from our refined database. There are 1154 sector crossings in Svalgaard's list in 1976-2016 and 893 in the refined list.

The annual numbers of Svalgaard's sector crossings and of the refined subset with more accurate timing (hereafter called refined HMF sector crossings) are shown in the third panel of Figure 1. The bottom panel shows the 13-month running mean of sunspot numbers for reference (from SIDC). As can be seen from Figure 1, the data gaps in OMNI data set from the mid-1980s up to 1994 (due to only one Earth-orbiting satellite, IMP-8, measuring the solar wind at this time) significantly reduced the number of HMF sector crossing times of the refined list. As discussed earlier, depending on the solar cycle phase, mostly two or four sectors are observed per solar rotation at 1AU. As depicted in the third panel of Figure 1 a typical annual number of sector crossings (according to Svalgaard's list) lies in the range of about 30 which indicates a dominant two-sector structure in a good agreement with the overall view of sectors depicted in the upper two panels. Note that the HMF coverage is almost complete since 1995, which 
slightly increases the number of crossings in Svalgaard's list and considerably in the refined list.

\subsection{Mapping from Earth to Sun}

The HMF sector crossings observed at $1 \mathrm{AU}$ are mapped back to the Sun to get the corresponding photospheric magnetic field structure at the expected source at time $t_{\text {sun }}=t_{s c}-\Delta t$, where $t_{\text {sun }}$ is the time when the solar wind left the Sun (hereafter called solar source time), $t_{s c}$ is the HMF sector crossing time at $1 \mathrm{AU}$ and $\Delta t$ is the transit time from the Sun to $1 A U$. We assume that the field projects roughly radially from the photosphere to the source surface. This is, obviously, a coarse simplification of the coronal field, which can be assumed to be approximately valid and only over a part of the solar cycle. In particular, it is not valid during solar minimum times, when most of the source surface field projects typically to the polar coronal holes at midto high latitudes. However, it is better valid during the more active times of the cycle when coronal holes are found at all latitudes, including the low latitudes of the Earth's ballistic footpoint.

We assume that the solar wind flows radially outward from source surface to $1 A U$ and use two different models for solar wind velocity (delay time). The first one is mapping the refined HMF sector crossings to the Sun using the observed solar wind speed (mean value of solar wind speed within \pm 36 hours from the sector crossing time). A constant speed of the solar wind from the Sun to $1 \mathrm{AU}$ gives a better match of HMF sector polarities between the Sun and 1AU, especially for the slow solar wind (Koskela, Virtanen, and Mursula, 2017). However, the sector crossings of Svalgaard's list are mapped using a constant transit time of 5 days (Svalgaard and Wilcox, 1976; Svalgaard, Hannah, and Hudson, 2011; Hudson, Svalgaard, and Hannah, 2014). A detailed comparison of the photospheric field pattern in solar cycle 23 (see section 5) shows that the field polarity pattern at the Hale boundary is slightly more clear for the first method, but the overall field patterns are fairly similar for the two methods over the course of the solar cycle.

Figure 2 shows the Carrington longitudes of the solar sources calculated for the two lists (and methods) of the HMF sector crossings (corresponding source times can be calculated from Carrington longitudes). The solar source times calculated by mapping the refined HMF sector crossings using the measured solar wind speed are shown at the top panel and those calculated using the 5-day mapping of Svalgaard's sector crossing list are shown at the bottom panel. As discussed above, due to data gaps in OMNI data, the number of crossings is reduced in the top panel of Figure 2, particularly during solar cycle 22. Still the two panels show a very similar longitude-time pattern of crossings, even during times when the OMNI data coverage is worst. Figure 2 shows, e.g., the dominance of the two-sector structure in the declining phase, especially clearly in 2003 - 2005 and very similarly in the two panels (see also Figure 1). The sectors observed at these times are also seen to migrate very similarly with respect to the Carrington rotation.

\subsection{Solar cycle phase division}

We divide the solar cycle into four phases (minimum, ascending, maximum and declining phases) using the list of sunspot minimum and maximum times determined 

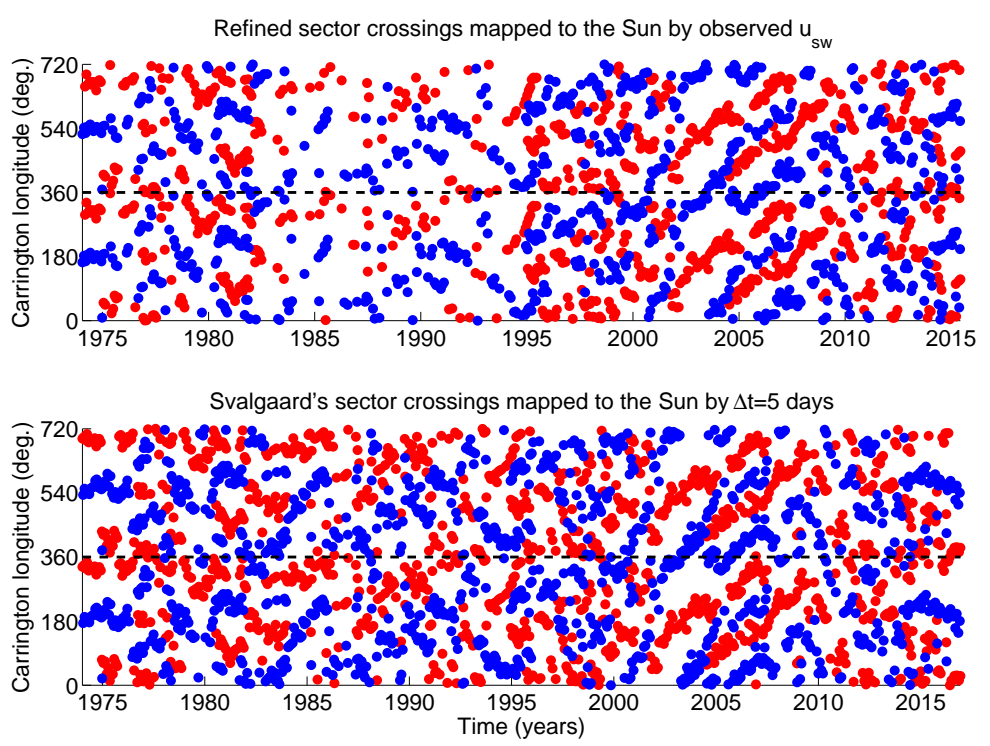

Figure 2. Carrington longitudes of the solar sources of HMF sector crossings. Top: crossings of the refined list mapped to the Sun by using observed solar wind speed $\left(u_{s w}\right)$; Bottom: Svalgaard's sector crossing list mapped using 5-day delay. The blue (red) color indicates the polarity change of sectors from positive (respectively, negative) to negative (positive). Note that the plots are replicated beyond the horizontal black dashed $360^{\circ}$ line for the sake of better visibility.

by the National Geophysical Data Center (ftp://ftp.ngdc.noaa.gov). For solar cycle 24 , the time for the sunspot maximum is not yet included in the list, and we choose 2014.5 as the maximum time for solar cycle 24 based on the prediction by SIDC (http://www.sidc.be). For each solar cycle, the date of the sunspot minimum is defined to have the phase of $0^{\circ}\left(360^{\circ}\right)$ and sunspot maximum $180^{\circ}$. We then divide the sunspot cycle into four phases linearly in time with a phase length of $90^{\circ}$ so that $0^{\circ}\left(360^{\circ}\right)$, $90^{\circ}, 180^{\circ}$ and $270^{\circ}$ will denote the centers of the minimum, ascending, maximum and declining phases of the solar cycle, respectively (Maliniemi, Asikainen, and Mursula, 2014). Then the ascending phase will be from $45^{\circ}$ to $135^{\circ}$, maximum phase from $135^{\circ}$ to $225^{\circ}$, declining phase from $225^{\circ}$ to $315^{\circ}$ and minimum phase from $315^{\circ}$ to $45^{\circ}$. The four phases of solar cycles 21-23 and the ascending and part of maximum phases of solar cycle 24 are shown in color in Figure 3. Note that the time span of the declining phase is greater than the ascending. It has long been known that most solar cycles have a shorter ascending than declining phase.

\subsection{Photospheric magnetic field}

The line-of-sight photospheric magnetic field is obtained from the daily level-3 magnetograms measured at the Wilcox Solar Observatory (WSO) from 1976 onwards. At WSO, the visible solar disk is imaged by using a 3 arc minute aperture instrument. Level-3 magnetograms are obtained every day using the Zeeman splitting of $525 \mathrm{~nm}$ 


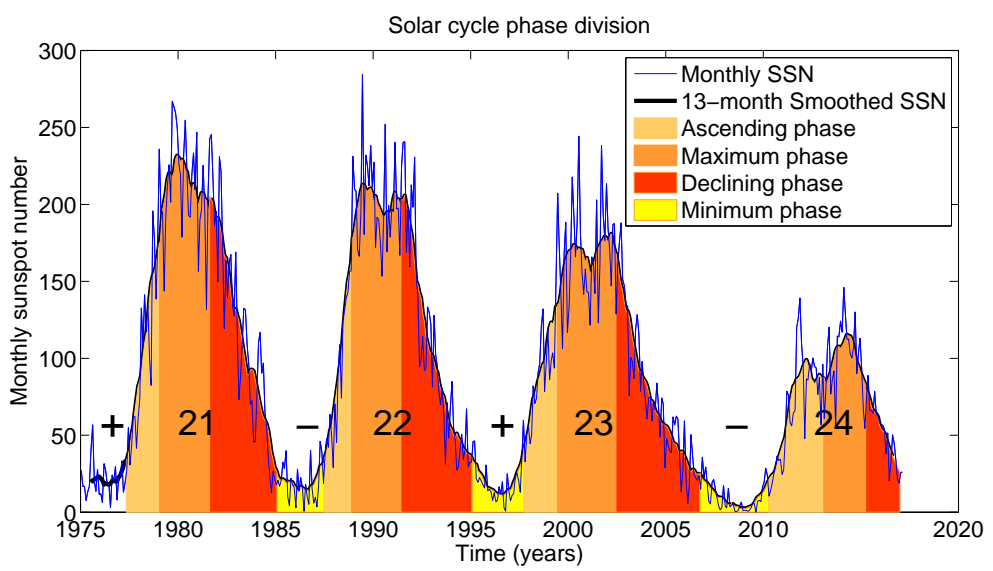

Figure 3. Phases of solar cycles $21-24$ denoted by color. Monthly and 13-month running mean sunspot numbers are denoted by thin blue and solid black lines. The $+(-)$ sign indicates the positive (negative) solar polarity minima.

Fe I line and remapped into the Carrington coordinate system binned into 23 longitude points within $\pm 55^{\circ}$ of longitude at $5^{\circ}$ resolution and 30 latitude points equally spaced within $\pm 14 / 15$ sine latitude. (Details of the data can be obtained at http://wso. stanford.edu/). For each HMF sector crossing the solar magnetogram corresponding to the calculated source day is selected, if available. If no source day magnetogram is available, magnetograms 1-2 days before or after the source day are selected and shifted in longitude. In the 2-day window, the magnetogram whose observation time is closest to the source time is selected. When shifting the data in longitude we take into account the fact that the Sun rotates about $13.2^{\circ}$ per day. Out of the 893 refined sector crossings in 1976-2016, 479 have a high-quality level-3 magnetogram in the calculated source day, and additional 308 in the 2-day window. So, only 106 sector crossings do not have a corresponding magnetogram within the 2-day window.

A superposed epoch analysis of several magnetograms is made for each cycle (solar cycles 21-24) and each phase of the solar cycle separately for $(-,+)$ and $(+,-)$ sector crossings. We only used magnetograms which were measured under good weather conditions, defined in the WSO terms with sky $\leq 2$. Note that at WSO, weather permitting, more than one magnetogram may be obtained per day. In such a case the magnetogram which is closest to the source time is selected from all sky $\leq 2$ magnetograms. 

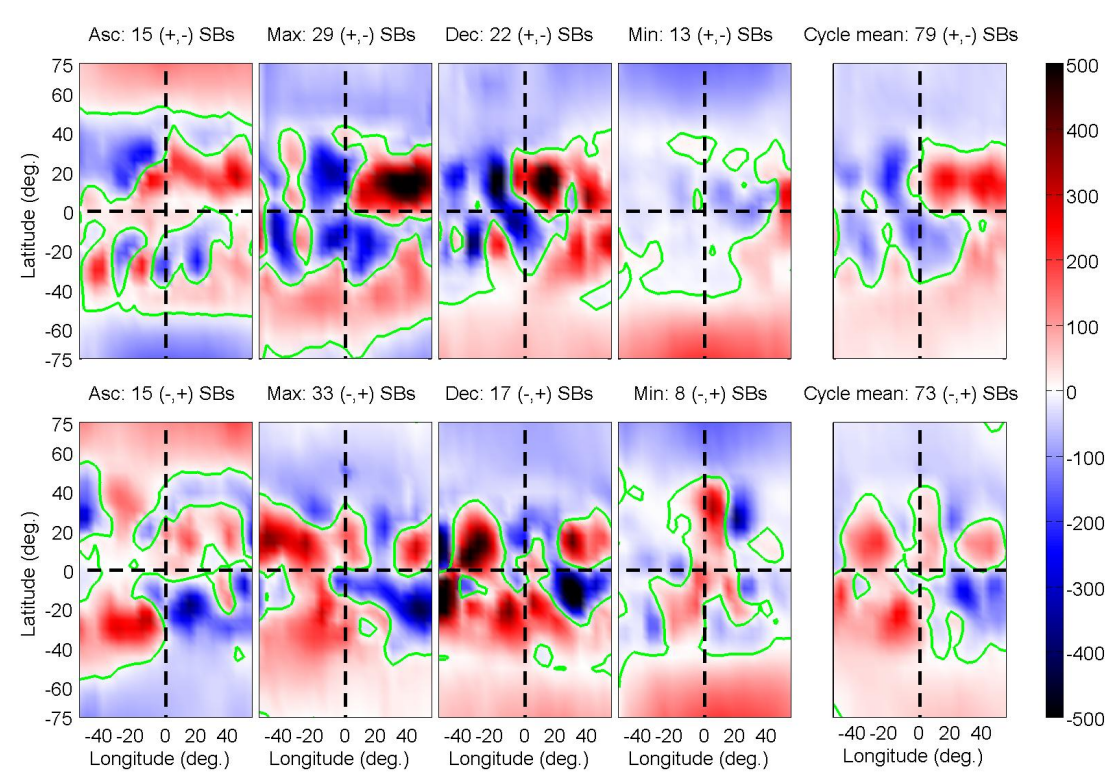

Figure 4. Structure of the line-of-sight photospheric magnetic field during refined HMF sector crossings in SC 21. Green curves represent the neutral line. Horizontal (vertical) dashed black lines indicate the equator (central meridian) of the Sun. Upper row gives results for $(+,-)$ crossings, lower row for $(-,+)$ crossings. Columns indicate ascending, maximum, declining, and minimum phases, and the overall cycle mean. Numbers of sector boundary crossings (SBs) are indicated in the title.

\section{HMF sector crossings in SC 21}

Figure 4 shows the structure of the average line-of-sight photospheric magnetic field during both the $(+,-)$ and $(-,+)$ sector crossings of the refined list, separately for the four phases of solar cycle 21 . The results during $(+,-)$ and $(-,+)$ sector crossings are shown in the first and second rows of Figure 4, respectively. The first four columns in both rows show the results for the ascending, maximum, declining and minimum phases, respectively, while the fifth column shows the averaged result for the whole solar cycle. The horizontal (vertical) black dashed lines denote the equator (central meridian) of the Sun. The title of each plot gives the number of sector crossings included in the plot. The blue color indicates negative (inward) polarity and red indicates positive (outward) polarity. The neutral line is represented by green color. In solar cycle 21 , a total of 79 $(+,-)$ and $73(-,+)$ HMF sector crossings are included to make the superposed epoch analysis of magnetograms, with somewhat different numbers of crossings included in the four cycle phases. Note that during the ascending (descending ) phase of odd solar cycles (e.g. cycle 21 and 23), the southern (northern) magnetic hemisphere is located in the northern high-latitude heliographic hemisphere and vice versa in the southern heliographic hemisphere. The situation is opposite during even solar cycles (e.g. cycle 22 and 24).

A boundary of oppositely oriented solar polarities close to the central meridian, when the order of sector crossing and the observed solar polarities match with the 
Hale polarity rule for sunspots of the current cycle and hemisphere, is called the Hale boundary (Svalgaard and Wilcox, 1976). If it is found in the opposite hemisphere, we call it anti-Hale boundary. We use the name Hale bipolar region hereafter to refer to such bipolar regions of oppositely oriented large scale solar polarities separated by the Hale boundary. By definition, a Hale bipolar region and Hale boundary always refer to a distribution within one hemisphere.

As the plots of the first row of Figure 4 show, the northern Hale boundary is evident during $(+,-)$ HMF sector crossings in all solar cycle phases except the minimum phase. However, the structure and location of the Hale bipolar regions are somewhat different for the three phases. During the ascending phase the Hale boundary is observed approximately at $+\left(20^{\circ}-30^{\circ}\right)$ latitude, partly shifted to negative longitude and the leading polarity is weighted to slightly lower latitudes than the trailing polarity. Note that this corresponds very well to the structure of a sunspot pair tilted in latitude according to Joy's law. On the other hand, there is no anti-Hale $(+,-)$ ordering of magnetic field polarities in the southern hemisphere. Rather, the central meridian is dominated by negative polarity, and a $(-,+)$ Hale polarity pattern of sectors is seen at $-25^{\circ}$ latitude and $-10^{\circ}$ longitude. The polarity of the polar magnetic field in the ascending phase is still same as in the preceding (positive polarity) minimum.

The northern Hale boundary in the maximum phase is also clearly developed. Note also that the positive polarity region is, on average, weighted toward a slightly lower latitude than in the ascending phase, in a good agreement with the decrease of sunspot latitudes during the solar cycle. (The higher latitudes of the northern hemisphere are already dominated by the new, negative polarity field). Again there is no (+,-) anti-Hale ordering of polarities in the southern hemisphere. The low latitudes of the southern hemisphere are even more strongly dominated by the negative polarity field than in the ascending phase, while at higher latitudes, there is a band of positive polarity field migrating poleward. These distributions are in agreement with the polarity and location of the new flux generated. Note that the southern polar magnetic field is not yet reversed in the maximum plot of Figure 4. This reflects the fact that the solar polar magnetic field reversal is often hemispherically asymmetric (e.g., Babcock, 1959; Makarov, Fatianov, and Sivaraman, 1983; Durrant and Wilson, 2003; Benevolenskaya, 2007; Virtanen and Mursula, 2010; Svalgaard and Kamide, 2013; Sun et al., 2015). Note also that, since solar cycle 20, the magnetic activity in the ascending phase is greater in the northern than southern hemisphere (e.g., Hiltula and Mursula, 2006; Temmer et al., 2006; Wang and Robbrecht, 2011; McIntosh et al., 2013), leading to an earlier reversal of the northern than southern polar magnetic field (e.g., Svalgaard and Kamide, 2013).

During the $(+,-)$ crossings of the declining phase, the northern hemisphere shows a closely similar structure to the maximum. The negative field covers now all longitudes before the Hale boundary so that the bipolar structure covers all longitudes included. The low latitudes of the southern hemisphere depict a less consistent dominance of negative polarity than at maximum, while the high latitudes show that the southern pole has finally reversed. Note also that, because of the earlier reversal of the northern pole, the negative polarity field takes a larger part of the whole area depicted in Figure 4. This is seen in the declining phase and even more clearly at minimum, and is related to the bashful ballerina phenomenon (Mursula and Hiltula, 2003), according to which the magnetic field dominant in the northern hemisphere extends over a larger region, thus leading to the southward shifted heliospheric current sheet. During the minimum 
phase the polar magnetic fields are strong, forming contiguous unipolar regions down to about $40^{\circ}$ in each hemisphere, with the northern pole extending slightly wider. It is obvious that the positive polarity sector observed at $1 \mathrm{AU}$ during the minimum has its source at mid to high latitudes of the southern hemisphere, while the negative polarity sector may come from either hemisphere and from a wider range of latitudes.

When averaged over the whole cycle (the fifth panel of Figure 4) the pattern of the photospheric field clearly reminds that of the maximum and declining phase. This is because of the larger number of crossings and the fairly similar structure during these two phases, as well as of the slightly larger intensity of the magnetic field at these times. The maximum intensity region of the leading polarity of the Hale bipolar structure is at a slightly lower latitude than the trailing polarity region. This is in agreement with the observed pattern of sunspots.

The second row of Figure 4 depicts the photospheric magnetic field patterns during the $(-,+)$ sector crossings when the Hale boundaries are expected to be found in the southern hemisphere. Over most of the cycle, the pattern is more complicated for $(-,+)$ crossings than for $(+,-)$ crossings, and the evidence for the Hale bipolar structure in the southern hemisphere is somewhat less systematic than for $(+,-)$ crossings in the northern hemisphere. This is also depicted in the cycle averaged panel, where the southern field remains partly latitudinally (not fully longitudinally) organized around the central meridian.

There is a Hale bipolar region in the southern hemisphere centered at about $-20^{\circ}$ latitude in the ascending phase. Note that the leading polarity region of the southern Hale bipolar structure is again at a lower (absolute) latitude than the trailing polarity region. Note also that the leading polarity region is connected to the polar region of the southern hemisphere which has the same polarity. The maximum phase and even the declining phase depict a partly latitudinal ordering of the southern field seen in the cycle mean plot. The Hale boundaries in the southern hemisphere are weakly seen during the maximum and declining phases.

During the minimum phase there is no clear Hale bipolar region in the southern hemisphere close to the central meridian. However, the overall dominance of the negative polarity, reflecting the wider extension of the northern polar region of the bashful ballerina structure, is repeated but less clearly than during $(+,-)$ crossings.

\section{HMF sector crossings in SC 22}

As mentioned earlier in section 2.1 and shown in Figures 1 and 2, due to the lack of observations of solar wind, there are several data gaps in OMNI data set especially during solar cycle 22. As a result, the number of sector crossings in the refined list is rather small in this cycle. Therefore, for this cycle, we used Svalgaard's sector crossing list mapped by the constant transit time of 5 days. Figure 5 shows the average photospheric magnetic field during Svalgaard's sector crossings for the four phases of cycle 22. The arrangement of panels of Figure 5 is the same as Figure 4, except that the order of $(+,-)$ and $(-,+)$ plots is changed in order to always depict the Hale ordered situation for the northern (southern) hemisphere in the top (bottom) panel. So, during cycle 22 (and other even cycles) the Hale pattern for $(-,+)$ crossings is in the northern hemisphere and for $(+,-)$ crossings in the south. In solar cycle 22, Svalgaard's list includes a total of 121 


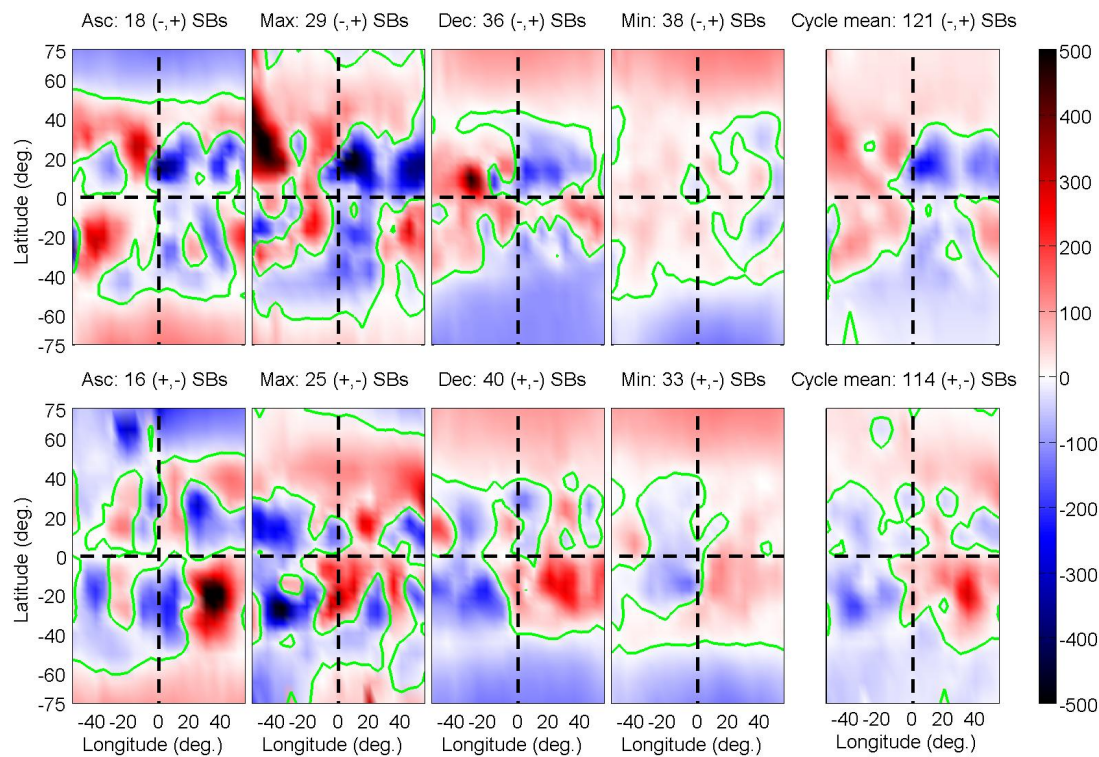

Figure 5. Same as Figure 4 but for the 5-day mapping of Svalgaard's HMF sector crossings in SC 22. Upper row gives now the results for $(-,+)$ crossings, lower row for $(+,-)$ crossings.

$(-,+)$ and $114(+,-)$ sector crossings, while for the refined list of would have been only $63(-,+)$ and $79(+,-)$ crossings.

Overall, the Hale pattern in the photospheric field at the central meridian is less systematic during solar cycle 22 than solar cycle 21 . However, during $(-,+)$ crossings a northern Hale bipolar region can be seen in the cycle mean (fifth panel) as well as in all other phases of the solar cycle except the minimum, at least weakly. Note that, during $(-,+)$ crossings in cycle 22 , there is evidence in the ascending and maximum phase for an anti-Hale boundary in the opposite hemisphere, so that the trailing polarity field may come from the southern hemisphere, rather than from the trailing part of the Hale bipolar region.

The second row of Figure 5 shows the field structure during (+,-) HMF sector crossings. In this case, there is evidence for Hale boundaries in the southern hemisphere during all phases (including the minimum) and the average plot. However, in the ascending phase and maximum, the Hale boundary is somewhat shifted in longitude. During (+,-) HMF sector crossings in cycle 22, there is also evidence for a weakly organized $(+,-)$ anti-Hale pattern in the north during the minimum phase.

\section{HMF sector crossings in SC 23}

Solar activity in cycle 23 was weaker than in the two preceding cycles and the polar magnetic fields were weaker (e.g, Wang, Robbrecht, and Sheeley, 2009; Hoeksema, 2010). Consequently, the heliospheric current sheet tilt was larger than typical in the 


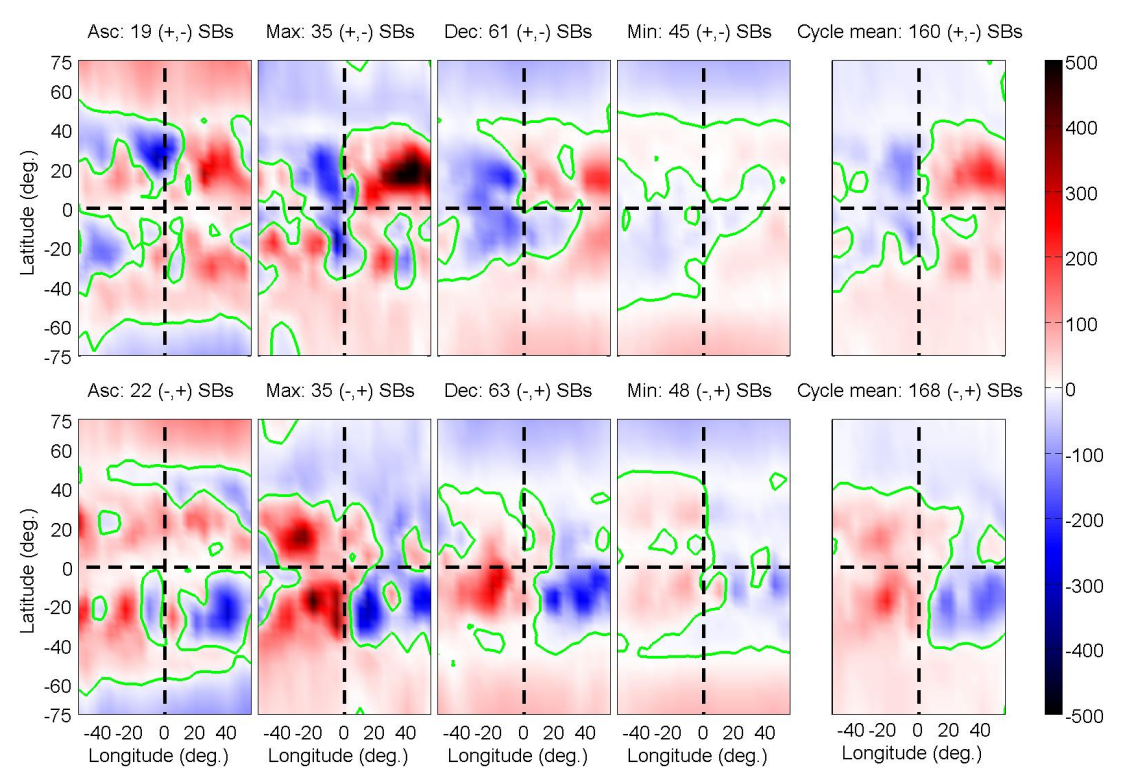

Figure 6. Same as Figure 4 but for solar cycle 23.

declining phase (e.g, Pishkalo, 2006; Wang, Robbrecht, and Sheeley, 2009; Virtanen and Mursula, 2010; Cliver, Richardson, and Ling, 2013), and the heliospheric magnetic field was weaker (e.g, Smith and Balogh, 2008). As it can be seen in Figure 1, the annual number of refined and Svalgaard's sector crossings in this cycle are almost equal, since the OMNI data set is almost complete. We now use both the refined sector crossings mapped back by the observed solar wind speed and Svalgaard's list using a constant transit time of 5 days to compare the field patterns between the two methods.

Figure 6 shows the structure of the photospheric magnetic field during refined sector crossings in SC 23. In this case, magnetograms include a total of $160(+,-)$ and $168(-,+)$ HMF sector crossings. The photospheric field pattern during $(+,-)$ crossings shows a very similar structure as during $(+,-)$ crossings in solar cycle 21 (see Figure 4 ), except that the field intensity is weaker in solar cycle 23. A clear Hale bipolar region is depicted in the north during $(+,-)$ HMF sector crossings in all other phases except the minimum phase. One can see a Hale boundary at about $+25^{\circ}$ latitude in the ascending phase and at slightly lower latitude of about $+15^{\circ}$ in the maximum and declining phase.

The second row of Figure 6 presents the field structure during $(-,+)$ crossings. There is now some similarity to the corresponding pattern of SC 21 , but less than for $(+,-)$ crossings. In particular, the field during SC 23 depicts more evidence for Hale pattern than SC 21. Indeed there is clear evidence for a Hale bipolar structure in the southern hemisphere during $(-,+)$ crossings in the maximum and declining phase, and more weakly in the ascending phase and minimum. The Hale patterns of the maximum, declining, and even the minimum phases are surprisingly similar. There is also evidence for weak anti-Hale structure in the north during the $(-,+)$ crossings especially in the declining and minimum phase. Similar to the $(+,-)$ crossings, also during the $(-,+)$ sector 


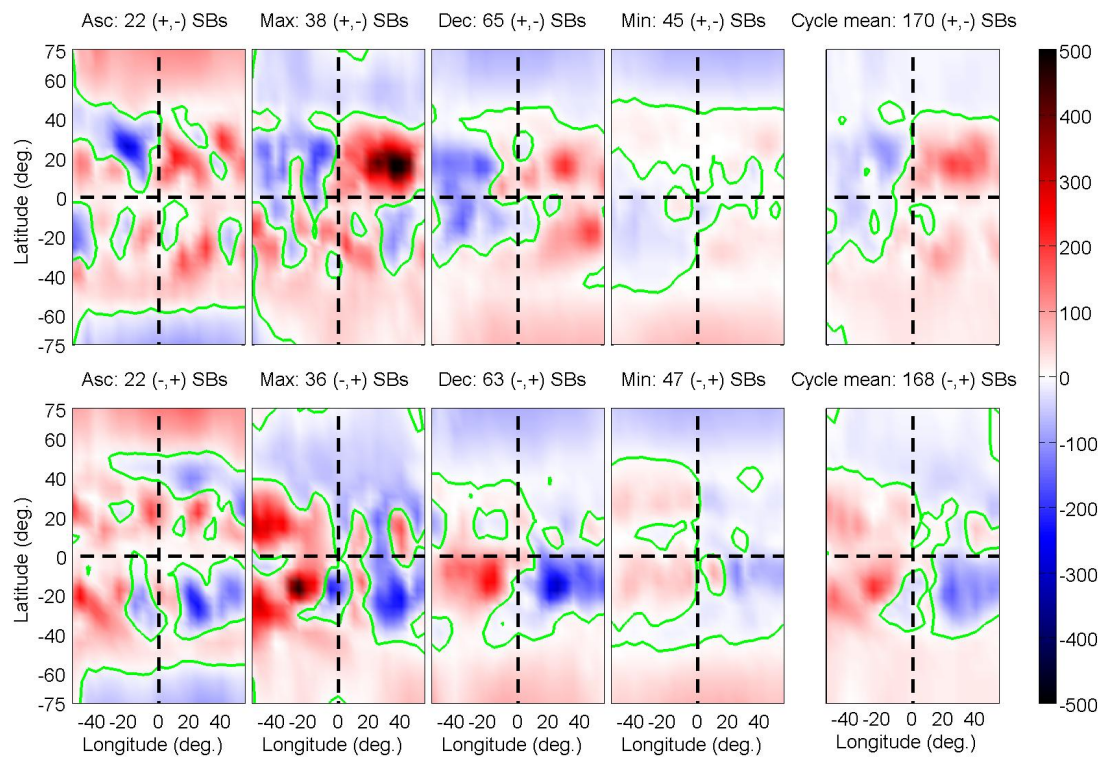

Figure 7. Same as Figure 6 except that here the 5-day mapping of Svalgaard's HMF sector crossings time is used.

crossings, the latitudinal position of the Hale bipolar region decreases as the solar cycle progresses (in agreement with Spörer's law) from the ascending to the declining phase.

Figure 7 depicts the field structure during the HMF sector crossings of the Svalgaard's list of solar cycle 23. Here, magnetograms include a total of $170(+,-)$ and $168(-,+)$ HMF sector crossings. During all phases of the solar cycle, the photospheric field distribution depicted in Figure 6 and Figure 7 are fairly similar especially in the Hale boundary hemisphere for both $(+,-)$ and $(-,+)$ crossings, but the field polarities are slightly more clearly ordered according to Hale boundaries at the central meridian in Figure 6. Moreover, in some cases (e.g. the overall plot in the south for $(-,+)$ crossings) the Hale boundary is shifted by about $-20^{\circ}$ in longitude in Figure 7 . This implies, that the 5-day mapping of Svalgaard's sector crossing list gives a reasonable approximation to the refined case but may be shifted in longitude roughly by 1 day.

\section{HMF sector crossings in SC 24}

Figure 8 depicts the photospheric field structure during the sector crossings of the refined list in SC 24. Since solar cycle 24 is not yet complete, we can present here the results for the ascending, maximum and early declining phase only (we include data until November 30, 2016 and assume that the next minimum will be in June 2020). In this case, there are in total $72(-,+)$ and $75(+,-)$ HMF sector crossings. As shown in the first row of Figure 8 , a Hale boundary is seen during $(-,+)$ crossings in the 

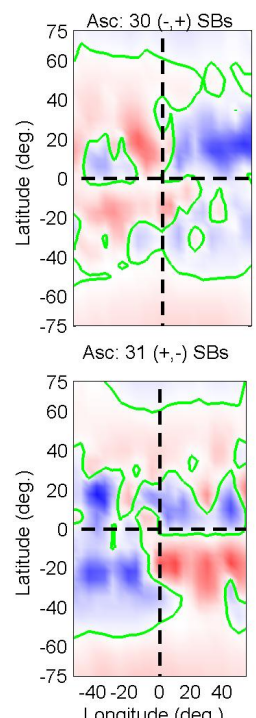

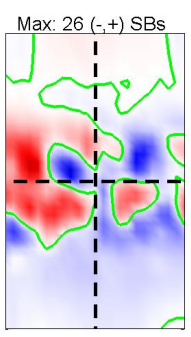

Max: $27(+,-)$ SBs

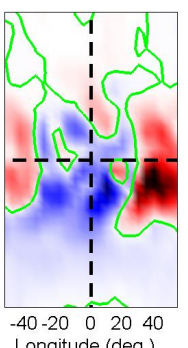

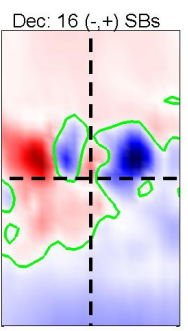

Dec: $17(+,-)$ SBs

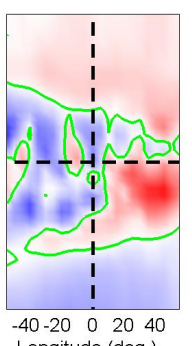

$\begin{array}{cccc}-40-20 & 0 & 20 & 40 \\ \text { Longitude (deg.) }\end{array}$

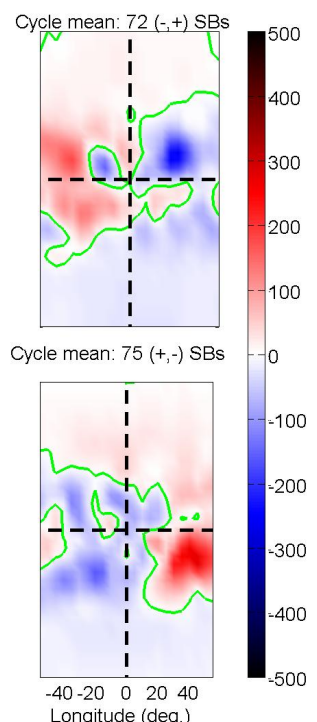

Longitude (deg.)

Figure 8. Same as Figure 5 but here for the ascending, maximum and declining phases of solar cycle 24.

north clearly in the ascending phase and weakly in the maximum and declining phase. Note that during the maximum time, the southern pole already has reversed but the northern reversal was still delaying. In the declining phase, the positive polarity field takes somewhat a larger part of the whole area depicted in Figure 8, indicating that the the bashful ballerina phenomenon of solar cycle 24 has already started.

The second row of Figure 8 depicts the field structure during the $(+,-)$ crossings. In this case, a Hale boundary at the central meridian is observed in the south clearly during the ascending and weakly in the declining phase. In the cycle mean plot (fourth panel), the Hale boundary is observed at about $+10^{\circ}$ longitude and in maximum even further in longitude.

\section{Significance test}

We made a statistical estimate of the significance of the observed Hale distributions by superposing randomly sampled magnetograms, and comparing their field strengths with those of the superposed magnetograms of the HMF crossings. The difference between the maximum and the minimum magnetic fields (hereafter called $\delta B_{\text {observed }}^{+-}$) of the superposed magnetograms of the $(+,-)$ HMF crossings and also the difference between the minimum and maximum fields (hereafter called $\delta B_{\text {observed }}^{-+}$) of the superposed magnetograms of the $(-,+)$ HMF crossings were first calculated. Then, magnetograms were randomly sampled from among all magnetograms available in the respective solar cycle phase. The number of randomly sampled magnetograms was taken to be the same as in the Hale boundary plots. The randomization process was repeated 100 times 

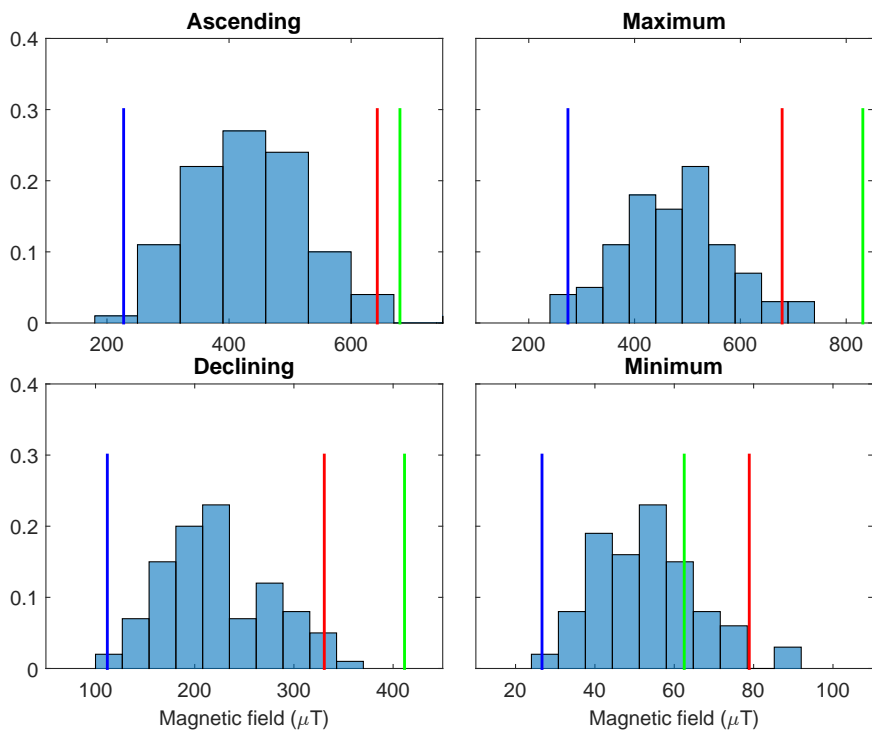

Figure 9. Histogram distribution of $\delta B^{+-} \quad$ during the ascending (top left panel), maximum (top right panel), declining (bottom left panel) and minimum (bottom right panel) phase of solar cycle 23. Blue and red vertical lines represent the lower and the upper boundaries of the $95 \%$ confidence level of the distribution, respectively. The green vertical line represents $\delta B_{\text {observed }}^{+-}$

for each ordering, hemisphere and cycle phase. For each superposed magnetogram, the difference between the maximum and minimum magnetic field (hereafter called $\left.\delta B_{\text {random }}^{+-}\right)$across a $(+,-)$ordered Hale boundary, or the difference between the minimum and maximum magnetic field (hereafter called $\delta B_{\text {random }}^{-+}$) across a $(-,+)$ordered Hale boundary was calculated for each hemisphere and solar cycle phase.

Figure 9 shows the distribution of $\delta B_{\text {random }}^{+-}$for the four phases of solar cycle 23 with lower and upper boundaries of the $95 \%$ confidence interval shown in blue and red vertical lines, respectively. The green vertical line represents $\delta B_{\text {observed }}^{+-}$for the northern hemisphere obtained from the top four panels (from left to right) of Figure 6. As can be seen from Figure 9, in the ascending (top left panel), maximum (top right panel) and declining phase (bottom left panel), the values of $\delta B_{\text {observed }}^{+-}$are beyond the $95 \%$ confidence interval of $\delta B_{\text {random }}^{+-}$. Accordingly, the values of $\delta B_{\text {observed }}^{+-}$are larger than $95 \%$ of the randomly selected superposed magnetograms in the three solar cycle phases of the northern hemisphere. This gives evidence that the superposed magnetograms formed from the HMF crossings indeed have a polarity pattern which is correctly ordered across the Hale boundary in a significant way. On the other hand, in the minimum phase (bottom right panel), the value of $\delta B_{\text {observed }}^{+-}$is insignificant (in between the $95 \%$ confidence intervals of $\delta B_{\text {random }}^{+-}$).

Figure 10 is the same as Figure 9, but for $\delta B^{-+}$. In this case, $\delta B_{\text {observed }}^{-+}$is obtained from the four bottom panels of Figure 6. As can be seen in Figure 10, in all phases the $\delta B_{\text {observed }}^{-+}$(green vertical line) is beyond the $95 \%$ confidence interval, indicating that 

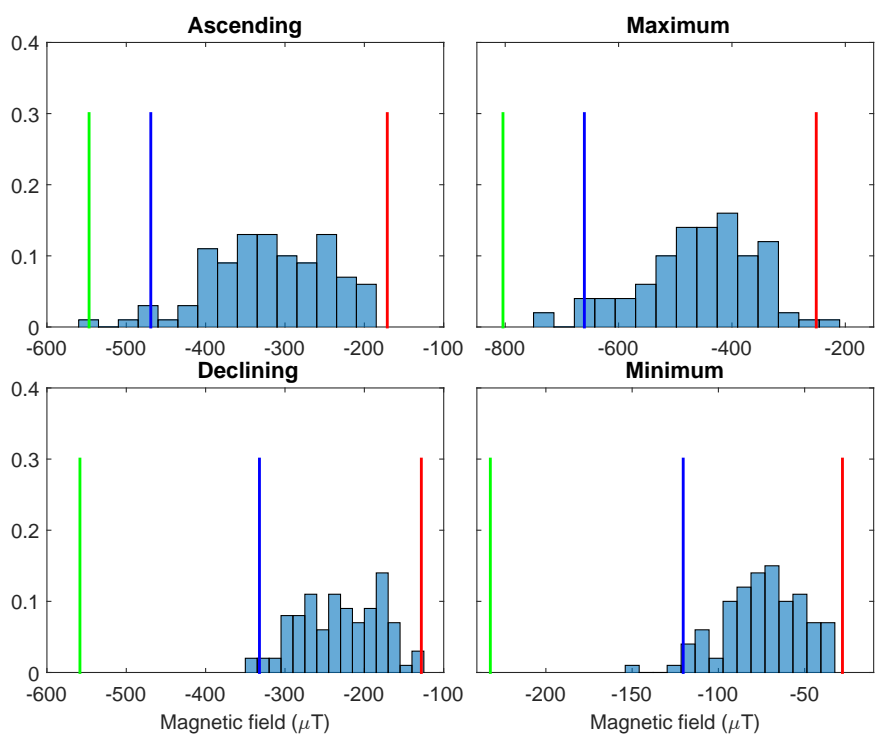

Figure 10. The same as Figure 9 but here the histogram distribution of $\delta B_{\text {random }}^{-+}$.

Hale boundaries for $(-,+)$ HMF crossings are statistically significant in the southern hemisphere for all phases of SC 23. This strongly supports the validity of our results and the validity of Hale boundaries.

\section{Discussion and conclusions}

In this paper, we have studied the structure of the photospheric magnetic field during HMF sector crossings in the four phases of solar cycles $21-24$. The HMF sector crossings observed at $1 \mathrm{AU}$ are ballistically mapped back to the Sun to determine the corresponding photospheric magnetic field structure at the source. As expected, the photospheric field structure observed during HMF sector crossings shows differences between the different solar cycle phases, corresponding to the evolution of the solar magnetic field over the solar cycle. More interestingly, we found that the field patterns during $(+,-)$ and $(-,+)$ crossings were generally quite different. This is mainly due to the appearance of strong Hale polarity regions that have opposite polarities and occur at opposite hemispheres for the $(+,-)$ and $(-,+)$ HMF crossings.

The occurrence of photospheric Hale and anti-Hale boundaries corresponding to the HMF sector crossings observed at $1 \mathrm{AU}$ during the four phases of solar cycles 21 24 are summarized in Table 1. Table 1 shows that Hale boundaries occur, at least weakly in all ascending, maximum and declining phases of the solar cycles studied in this paper. On the other hand, there is evidence for weak Hale boundaries during only two minimum times out of six possible cases. The Hale pattern in the north is clear in eight out of fifteen possible cases, but only five cases in the south. Overall, the field 
Table 1. Occurrence of the photospheric Hale boundary corresponding to HMF sector crossings. The first and second columns show the solar cycle and corresponding cycle phases, the third and fourth columns indicate the occurrence of $(+,-)$ photospheric field pattern during $(+,-)$ HMF sector crossings and the fifth and sixth columns show the $(-,+)$ photospheric field pattern during $(-,+)$ HMF sector crossings. Note that texts highlighted with red color are the possible Hale patterns in that solar cycle and those which are statistically significant are marked as $*$.

\begin{tabular}{|c|c|c|c|c|c|}
\hline Solar cycle & Phase & $\begin{array}{c}(+,-) \text { field } \\
\text { pattern (north) }\end{array}$ & $\begin{array}{c}(+,-) \text { field } \\
\text { pattern (south) }\end{array}$ & $\begin{array}{c}(-,+) \text { field } \\
\text { pattern (north) }\end{array}$ & $\begin{array}{c}(-,+) \text { field } \\
\text { pattern (south) }\end{array}$ \\
\hline \multirow[t]{4}{*}{21} & Ascending & clear & none & none & clear* \\
\hline & Maximum & clear* & none & unclear & weak \\
\hline & Declining & clear* & unclear & none & weak* \\
\hline & Minimum & unclear & none & unclear & unclear \\
\hline \multirow[t]{4}{*}{22} & Ascending & unclear & weak* & weak* & weak \\
\hline & Maximum & unclear & weak & clear* & weak \\
\hline & Declining & unclear & clear * & weak* & none \\
\hline & Minimum & weak & weak* & unclear & none \\
\hline \multirow[t]{4}{*}{23} & Ascending & clear ${ }^{*}$ & none & none & weak* \\
\hline & Maximum & clear* & unclear & unclear & clear* \\
\hline & Declining & clear* & none & weak & clear* \\
\hline & Minimum & none & weak & weak & weak* \\
\hline \multirow[t]{3}{*}{24} & Ascending & none & clear* & clear & unclear \\
\hline & Maximum & none & weak * & weak* & none \\
\hline & Declining & none & weak & weak* & unclear \\
\hline
\end{tabular}

polarity structure in the southern hemisphere is found to be slightly more complex than in the north, and there is slightly less clear evidence for Hale boundaries in the southern hemisphere. This is particularly true for solar cycle 21 , where the northern hemisphere depicted clear Hale pattern for $(+,-)$ crossings in the three cycle phases but the southern hemisphere appeared to be partly ordered in latitude than in longitude and depicted the Hale boundaries less clearly.

Using the method discussed in Section 7, we made a statistical estimate of the significance of the observed Hale distributions. The statistical estimates strongly support the validity of the Hale boundaries especially in the maximum and declining phase of the solar cycle. The statistically significant Hale boundaries are marked as $*$ in Table 1 . Accordingly, six out of the eight Hale boundaries observed in the ascending phase are statistically significant. The same result is valid for the solar maxima. In the declining phase even seven Hale boundaries are statistically significant. The Hale patterns that exist in the two minima are both significant.

The Hale pattern is found to be more systematic during the $(+,-)$ crossings of the odd solar cycles (solar cycles 21 and 23) in the north than during the $(-,+)$ crossing of the even solar cycle 22 in the north. Moreover, the photospheric field pattern during solar cycle 23 shows a closely similar structure as during solar cycle 21 especially for the north during $(+,-)$ crossings, except that the field intensity is weaker in solar cycle 23. On the other hand, the Hale structure of even cycles (solar cycle 22 and 24) shows less of mutual resemblance in either hemisphere.

We found that the latitudinal position of the Hale bipolar regions observed in this paper occasionally decreases as the the solar cycle progresses from the ascending to 
declining phase, which is in agreement with Spörer's law (see Spörer and Maunder, 1890). The fact that Hale bipolar regions show similar property with sunspots supports the idea that sunspots and Hale boundaries are linked to each other. It has also been shown earlier that solar active regions (sunspots and solar flares) appear preferentially at Hale boundaries (see e.g., Dittmer, 1975; Svalgaard, Hannah, and Hudson, 2011; Hudson, Svalgaard, and Hannah, 2014; Akasofu, 2014, 2015). However, the question of why solar active regions appear preferentially at Hale boundaries requires further investigation (see e.g., Hudson, Svalgaard, and Hannah, 2014). The warps in HCS, for example at $1 \mathrm{AU}$, are assumed to predominantly originate from the Hale boundaries at the photospheric level (e.g. Svalgaard, Hannah, and Hudson, 2011). The results presented in this paper show that the HMF sector crossings observed at $1 \mathrm{AU}$ have at least a partial correspondence to Hale boundaries in the photosphere.

Solar magnetic activity shows north-south asymmetric behavior, i.e, the northern and southern hemispheres most of the time do not show equal magnetic activity. For example, polar fields typically reverse at different times. In recent cycles the northern hemisphere often reversed ahead of the south (e.g., Makarov, Fatianov, and Sivaraman, 1983; Durrant and Wilson, 2003; Benevolenskaya, 2007; Virtanen and Mursula, 2010; Svalgaard and Kamide, 2013; Sun et al., 2015). Moreover, during roughly three years in the declining to minimum phase of all studied solar cycles, the southern hemisphere has been more active than the north (e.g. Zhao, Hoeksema, and Scherrer, 2005; Norton and Gallagher, 2010; Wang and Robbrecht, 2011; McIntosh et al., 2013; Virtanen and Mursula, 2014). A stronger magnetic intensity of the field dominant in the southern hemisphere implies, by the conservation of magnetic flux, a weaker but more widely spread field of the northern hemisphere. This leads to the HCS being slightly (on an average by $+2^{\circ}$ ) but persistently shifted southwards (Mursula and Hiltula, 2003; Virtanen and Mursula, 2010; Erdös and Balogh, 2010), i.e. to the phenomenon called the bashful ballerina. The photospheric field polarity distribution presented in this paper verifies the larger area of the field of the northern hemisphere during the declining and minimum phases, in a good agreement with the bashful ballerina phenomenon.

Acknowledgements We acknowledge the financial support by the Academy of Finland to the ReSoLVE Centre of Excellence (project no. 272157). Wilcox Solar Observatory data used in this study was obtained via the web site http://wso.stanford.edu courtesy of J.T. Hoeksema. The OMNI data were obtained from the GSFC/SPDF OMNIWeb interface at http://omniweb.gsfc.nasa.gov. We thank L. Svalgaard for the HMF polarity data. We are also grateful to National Geophysical Data Center and WDCSILSO, Royal Observatory of Belgium, Brussels for sunspot data.

Disclosure of Potential Conflicts of Interest: The authors declare that they have no conflicts of interest.

\section{References}

Akasofu, S.-I.: 2014, Single spots, unipolar magnetic regions, and pairs of spots. Geophys. Res. Lett. 41, 3698. DOI. ADS. 
Akasofu, S.-I.: 2015, Single spots, unipolar magnetic regions, and pairs of spots: 2. The development of sunspot pairs and the Hale boundary. Geophys. Res. Lett. 42, 2571. DOI. ADS.

Antonucci, E., Svalgaard, L.: 1974, Green Corona and Solar Sector Structure. Solar Phys. 36, 115. DOI. ADS.

Babcock, H.D.: 1959, The Sun's Polar Magnetic Field. Astrophys. J. 130, 364. DOI. ADS.

Balogh, A., Erdös, G.: 2013, The Heliospheric Magnetic Field. Space Sci. Rev. 176, 177. DOI. ADS.

Benevolenskaya, E.E.: 2007, Polar magnetic field reversals on the Sun. Highlights of Astronomy 14, 273. DOI. ADS.

Cliver, E.W., Richardson, I.G., Ling, A.G.: 2013, Solar Drivers of 11-yr and Long-Term Cosmic Ray Modulation. Space Sci. Rev. 176, 3. DOI. ADS.

Crooker, N.U., Lazarus, A.J., Phillips, J.L., Steinberg, J.T., Szabo, A., Lepping, R.P., Smith, E.J.: 1997, Coronal streamer belt asymmetries and seasonal solar wind variations deduced from Wind and Ulysses data. J. Geophys. Res. 102, 4673. DOI. ADS.

Dittmer, P.H.: 1975, The relationship between solar flares and solar sector boundaries. Sol. Phys. 41, 227. DOI. ADS.

Durrant, C.J., Wilson, P.R.: 2003, Observations and Simulations of the Polar Field Reversals in Cycle 23. Sol. Phys. 214, 23. DOI. ADS.

Erdös, G., Balogh, A.: 2010, North-south asymmetry of the location of the heliospheric current sheet revisited. J. Geophys. Res. (Space Physics) 115, A01105. DOI. ADS.

Hiltula, T., Mursula, K.: 2006, Long dance of the bashful ballerina. Geophys. Res. Lett. 33, L03105. DOI. ADS.

Hoeksema, J.T.: 2010, Evolution of the large-scale magnetic field over three solar cycles. In: Kosovichev, A.G., Andrei, A.H., Rozelot, J.-P. (eds.) IAU Symposium, IAU Symposium 264, 222. DOI. ADS.

Hoeksema, J.T., Wilcox, J.M., Scherrer, P.H.: 1983, The structure of the heliospheric current sheet 1978-1982. J. Geophys. Res. 88, 9910. DOI. ADS.

Hudson, H.S., Svalgaard, L., Hannah, I.G.: 2014, Solar Sector Structure. Space Sci. Rev. 186, 17. DOI. ADS.

Koskela, J.S., Virtanen, I.I., Mursula, K.: 2017, Comparing Coronal and Heliospheric Magnetic Fields over Several Solar Cycles. Astrophys. J. 835, 63. DOI. ADS.

Makarov, V.I., Fatianov, M.P., Sivaraman, K.R.: 1983, Poleward migration of the magnetic neutral line and the reversal of the polar fields on the sun. I - Period 1945-1981. Sol. Phys. 85, 215. DOI. ADS.

Maliniemi, V., Asikainen, T., Mursula, K.: 2014, Spatial distribution of Northern Hemisphere winter temperatures during different phases of the solar cycle. J. Geophys. Res. (Atmospheres) 119, 9752. DOI. ADS.

Mansurov, S.M.: 1970, New Evidence of a Relationship between Magnetic Fields in Space and on Earth. Geomagn. Aeron. 9, 622. ADS.

McIntosh, S.W., Leamon, R.J., Gurman, J.B., Olive, J.-P., Cirtain, J.W., Hathaway, D.H., Burkepile, J., Miesch, M., Markel, R.S., Sitongia, L.: 2013, Hemispheric Asymmetries of Solar Photospheric Magnetism: Radiative, Particulate, and Heliospheric Impacts. Astrophys. J. 765, 146. DOI. ADS.

Mursula, K., Hiltula, T.: 2003, Bashful ballerina: Southward shifted heliospheric current sheet. Geophys. Res. Lett. 30(22), 2135. DOI. ADS.

Mursula, K., Hiltula, T.: 2004, Systematically Asymmetric Heliospheric Magnetic Field: Evidence for a Quadrupole Mode and Non-Axisymmetry with Polarity Flip-Flops. Sol. Phys. 224, 133. DOI. ADS.

Mursula, K., Virtanen, I.: 2011, The last dance of the bashful ballerina? AEFA 525, L12. DOI. ADS.

Mursula, K., Zieger, B.: 2001, Long-term north-south asymmetry in solar wind speed inferred from geomagnetic activity: A new type of century-scale solar oscillation? Geophys. Res. Lett. 28, 95. DOI. ADS.

Mursula, K., Hiltula, T., Zieger, B.: 2002, Latitudinal gradients of solar wind speed around the ecliptic: Systematic displacement of the streamer belt. Geophys. Res. Lett. 29, 28. DOI. ADS.

Norton, A.A., Gallagher, J.C.: 2010, Solar-Cycle Characteristics Examined in Separate Hemispheres: Phase, Gnevyshev Gap, and Length of Minimum. Sol. Phys. 261, 193. DOI. ADS.

Norton, A.A., Charbonneau, P., Passos, D.: 2014, Hemispheric Coupling: Comparing Dynamo Simulations and Observations. Space Sci. Rev. 186, 251. DOI. ADS

Pishkalo, M.I.: 2006, Reconstruction of the Heliospheric Current Sheet Tilts Using Sunspot Numbers. Solar Phys. 233, 277. DOI. ADS.

Rosenberg, R.L., Coleman, P.J. Jr.: 1969, Heliographic latitude dependence of the dominant polarity of the interplanetary magnetic field. J. Geophys. Res. 74, 5611. DOI. ADS.

Sanderson, T.R., Appourchaux, T., Hoeksema, J.T., Harvey, K.L.: 2003, Observations of the Sun's magnetic field during the recent solar maximum. J. Geophys. Res. (Space Physics) 108, 1035. DOI. ADS.

Schulz, M.: 1973, Interplanetary sector structure and the helliomagnetic equator. Astrophys. Space Sci. 24, 371. DOI. ADS. 
Simpson, J.A., Zhang, M., Bame, S.: 1996, A Solar Polar North-South Asymmetry for Cosmic-Ray Propagation in the Heliosphere: The ULYSSES Pole-to-Pole Rapid Transit. Astrophys. J. Lett. 465, L69. DOI. ADS.

Smith, E.J., Balogh, A.: 2008, Decrease in heliospheric magnetic flux in this solar minimum: Recent Ulysses magnetic field observations. Geophys. Res. Lett. 35, L22103. DOI. ADS.

Smith, E.J., Jokipii, J.R., Kóta, J., Lepping, R.P., Szabo, A.: 2000, Evidence of a North-South Asymmetry in the Heliosphere Associated with a Southward Displacement of the Heliospheric Current Sheet. Astrophys. J. 533, 1084. DOI. ADS.

Spörer, F.W.G., Maunder, E.W.: 1890, Prof. Spörer's researches on Sun-spots. Mon. Not. Roy. Astron. Soc. 50, 251. DOI. ADS.

Sun, X., Hoeksema, J.T., Liu, Y., Zhao, J.: 2015, On Polar Magnetic Field Reversal and Surface Flux Transport During Solar Cycle 24. Astrophys. J. 798, 114. DOI. ADS.

Svalgaard, L.: 1968, Sector structure of the interplanetary magnetic field and daily variation of the geomagnetic field at high latitudes. Geophys. papers r-6, Danish Met. Inst., Copenhagen, Denmark.

Svalgaard, L., Kamide, Y.: 2013, Asymmetric Solar Polar Field Reversals. Astrophys. J. 763, 23. DOI. ADS.

Svalgaard, L., Wilcox, J.M.: 1976, The Hale solar sector boundary. Sol. Phys. 49, 177. DOI. ADS.

Svalgaard, L., Hannah, I.G., Hudson, H.S.: 2011, Flaring Solar Hale Sector Boundaries. Astrophys. J. 733, 49. DOI. ADS.

Temmer, M., Rybák, J., Bendík, P., Veronig, A., Vogler, F., Otruba, W., Pötzi, W., Hanslmeier, A.: 2006, Hemispheric sunspot numbers $\left\{\mathrm{R}_{n}\right\}$ and $\left\{\mathrm{R}_{s}\right\}$ from 1945-2004: catalogue and N-S asymmetry analysis for solar cycles 18-23. AE A 447, 735. DOI. ADS.

Virtanen, I.I., Mursula, K.: 2010, Asymmetry of solar polar fields and the southward shift of HCS observed by Ulysses. J. Geophys. Res. (Space Physics) 115, A09110. DOI. ADS.

Virtanen, I.I., Mursula, K.: 2014, North-South Asymmetric Solar Cycle Evolution: Signatures in the Photosphere and Consequences in the Corona. Astrophys. J. 781, 99. DOI. ADS.

Vokhmyanin, M.V., Ponyavin, D.I.: 2013, Sector structure of the interplanetary magnetic field in the nineteenth century. Geophys. Res. Lett. 40, 3512. DOI. ADS.

Wang, Y.-M., Robbrecht, E.: 2011, Asymmetric Sunspot Activity and the Southward Displacement of the Heliospheric Current Sheet. Astrophys. J. 736, 136. DOI. ADS.

Wang, Y.-M., Robbrecht, E., Sheeley, N.R. Jr.: 2009, On the Weakening of the Polar Magnetic Fields during Solar Cycle 23. Astrophys. J. 707, 1372. DOI. ADS.

Wilcox, J.M., Howard, R.: 1968, A Large-Scale Pattern in the Solar Magnetic Field. Sol. Phys. 5, 564. DOI. ADS.

Wilcox, J.M., Ness, N.F.: 1965, Quasi-Stationary Corotating Structure in the Interplanetary Medium. J. Geophys. Res. 70, 5793. DOI. ADS.

Zhao, X.P., Hoeksema, J.T., Scherrer, P.H.: 2005, Prediction and understanding of the north-south displacement of the heliospheric current sheet. J. Geophys. Res. 110, 10101. DOI. ADS.

Zieger, B., Mursula, K.: 1998, Annual variation in near-Earth solar wind speed: Evidence for persistent north-south asymmetry related to solar magnetic polarity. Geophys. Res. Lett. 25, 841. DOI. ADS. 